\title{
PCR-based quarantine procedure for the detection of cocoa swollen shoot virus
}

\author{
A.K. Quainoo \\ Department of Biotechnology, University for Development Studies, Tamale, Ghana \\ Email:aquainoo@googlemail.com \\ ABSTRACT
}

\begin{abstract}
Cocoa (Theobroma cacao) is an economic tree crop which dominates the economy of most West African countries. Cocoa swollen shoot virus (CSSV) a badnavirus, transmitted by the mealy bug is a threat to the cocoa industry. Traditionally, indexing of cocoa trees for CSSV was usually carried out by budwood grafting donor materials onto disease-free Amelonado cocoa trees with symptoms of the virus expressed on the second or third leaf flushes. In CSSV budwood grafted transmissions involving genotypes other than Amelonado, symptoms may fail to develop on the leaves although the recipient trees may be infected. PCR/capillary electrophoresis was sensitive in detecting the CSSV and highlighted the disease well in advance of the appearance of visual symptoms. The design of the primer across the six published sequences of the cocoa genome capable of detecting all strains of the CSSV has the potential of improving the robustness of quarantine procedures for the international exchange and long term conservation of cocoa germplasm.
\end{abstract}

Keywords: Budwood grafting, cocoa, CSSV, genotypes, and PCR/capillary electrophoresis.

\section{INTRODUCTION}

Mealy bug transmitted cocoa swollen shoot virus disease (CSSVD) caused by the cocoa swollen shoot virus (CSSV) is mainly restricted to West Africa where it has resulted in the loss of several million of cocoa trees (Thresh, 1980). The CSSV belongs to the family Caulimoviridae and genus Badnavirus of which many distinct variants can be recognized usually named after the locality of their origin (Frison et al., 1999). The badnaviruses belong to the family Pararetroviridae now Badnaviridae. The CSSV particles are bacilliform and measure 121-130 x 28 $\mathrm{nm}$ (Frison and Feliu, 1989). The genome consists of double stranded DNA, circular with a total genome size of $7.4 \mathrm{~kb}$. CSSV is known to have latent infection which presents the greatest problem because the latent period is very variable and ranges from a few weeks to more than two years (Posnette, 1947).

Studies of CSSVD over the years have shown that diagnostic symptoms are not always manifested on the affected cocoa trees. Healthy cocoa trees which become infected may not show any symptoms for a considerable period of time. Removal of infected cocoa trees can prove difficult because trees in the latent period of infection are not always evident. Length of the latency period is influenced by the interval between inoculation, subsequent leaf growth flushes, the size of the plant, condition of the tree, age of the tree and the viral strain (Posnette, 1947). Thresh (1958), and Owusu and Bonney (1972) have shown that mealy bugs responsible for the transmission of the CSSV disease can acquire the virus from infected trees during the latter part of the latent period.

In Ghana, Nigeria and Togo virulent and mild strains of CSSV are known to occur with severe strains killing cocoa within two years (Posnette, 1947; Muller and Sackey, 2005). The non virulent strains predominates and cause various types of leaf chlorosis, root necrosis, root and stem swelling, and dieback in Amelonado cocoa trees in Ghana. The virulent strains are not lethal and rarely induce leaf symptoms although swellings are often pronounced (Legg, 1972).

The most common method of CSSVD diagnoses is based on visual inspection of indicator plants for symptoms of the disease. While this method is reliable and sensitive enough to detect CSSV symptoms, it is slow and inadequate for detecting latent infections. Recently, new molecular methods have become available for the detection of viruses in plants. Unlike visual inspection for disease symptoms on affected trees, these new methods are capable of identifying and quantifying the viral load even in part of the plant that appear healthy (Quainoo et al., 2008a and Quainoo et al., 2008b).

One of the most sensitive methods for CSSV detection currently available for cocoa is based on Polymerase Chain Reaction (PCR) (Quainoo et al., 
2008a and Quainoo et al., 2008b). PCR refers to the process of selective amplification of a small part of genetic code of a particular organism and this makes it possible to detect viral pathogens because each virus has its own unique genetic code.

Various primers for the detection of the CSSV using PCR has been designed (Muller. 2008; Cilas et al., 2005 and Hagen et al. 1993). However, it is important to avoid doubts over the identity of the CSSV strains affecting cocoa for international germplasm distribution of cocoa materials. The aim of this study was to design a primer capable of detecting all strains of the CSSV and identify the specific CSSV strains (through sequencing) affecting cocoa. This would lead to a PCR-based quarantine procedure for the detection of CSSV in cocoa trees and plantlets. The outcome of this research would improve the robustness of quarantine procedures for the international exchange and conservation of cocoa germplasm.

\section{MATERIALS AND METHODS}

The experiment was conducted in insect-proof cages within the University of Reading, UK glasshouses. CSSV (strain 1A) infected Amelonado cocoa tree (T2) and CSSV (strain 1A) infected cocoa genotype (SCA 6) were the source of budwoods for the experiment. Three CSSV-free cocoa clones (genotype CC 222, CRU 100 and CL 19/10) 1m high and nine CSSV-free somatic embryo derived plantlets (genotypes: 1=AMAZ 12, 2=EET272, 3=EET 272, 4=IMC 14, 5=LCTEEN 16/100, 6= SCA $3,7=$ SCA $6,8=$ SPA 4 and 9=SPA 4) 24 to 27 months old, were the experimental materials used.

Bdwood graft transmission of CSSV in cocoa clones: Budwoods from a CSSV (strain 1A) infected Amelonado cocoa tree (T2) were grafted to the lower portion of the stems ( $10 \mathrm{~cm}$ above soil level) of cocoa clones CC 222, CRU 100 and CL 19/10 with two branches each. Each clone was replicated two times. After budwood grafting new leaf flushes from the two branches on each cocoa clone were regularly observed for visual symptoms of CSSV (Figure 1). New leaf flushes were labeled and sampled using discs punched with $0.5 \mathrm{ml}$ eppendorf tube lids by inserting a leaf across an opened eppendorf tube and closing the tube to harvest equal size leaf samples (one for each leaf) as described by Quainoo et al., (2008a). The target region for leaf sampling was the minor vein regions of the laminar of each leaf every 30 days for 120 days. The new leaves were between $24 \mathrm{~cm}$ and $76 \mathrm{~cm}$ from the point of grafting on the cocoa clones. The sampled leaves were stored at -22
${ }^{\circ} \mathrm{C}$ for DNA extraction.

Bdwood graft transmission of CSSV in cocoa plantlets: Budwoods from CSSV (strain 1A) infected cocoa genotype (SCA 6) were grafted to the lower portion of the stems $(10 \mathrm{~cm}$ above soil level) of nine CSSV-free somatic embryo derived plantlets (genotypes: 1=AMAZ 12, 2=EET 272, 3=EET 272, 4=IMC 14, 5=LCTEEN 16/100, 6= SCA 3, 7=SCA 6, $8=$ SPA 4 and 9=SPA4) with two branches each. Mature leaves (ten) on each plantlet were labeled and sampled before budwood grafting, daily for one week and then every week up to six months post grafting. All new leaf flushes on each plantlet were labeled, observed for CSSV symptoms, sampled (as described above) every week up to six months post grafting. The new leaves were between $21 \mathrm{~cm}$ and 87 $\mathrm{cm}$ from the point of grafting on the cocoa plantlets. The sampled leaves were stored at $-22{ }^{\circ} \mathrm{C}$ for DNA extraction.

DNA extraction: Total genomic DNA was extracted from 20 sources: CSSV (strain 1A) infected Amelonado cocoa tree (T2), three virus-free cocoa clones (genotype CC 222, CRU 100 and CL 19/10), CSSV (strain 1A) infected cocoa genotype (SCA 6) and nine virus-free somatic embryo derived plantlets (1=AMAZ 12, 2=EET 272, 3=EET 272, 4=IMC 14, 5=LCTEEN 16/100, 6= SCA 3, 7=SCA 6, 8=SPA 4 and $9=S P A 4)$. A slightly modified DNeasy TM 96 Plant kit (Qiagen Ltd., UK) protocol was used. 400 $\mu \mathrm{l}$ of lysate Buffer AP1 (preheated to $65^{\circ} \mathrm{C}$ ) to dissolve and $2 \mu$ l of RNase were added to the eppendorf tubes containing the experimental materials and shaken with a tungsten pellet on a Retsch disrupter for 1.4 $\min \times 2$ at 25 cycles $s 1$. DNA extraction quality from the experimental materials was checked on an ethidium bromide-stained agarose gel.

CSSV primer design and PCR amplification: Primers for the CSSV were designed in the conserved regions of the six published sequences of the CSSV genome available from the National Center for Biotechnology Information database (NCBI) (AJ608931, AJ609019, AJ609020, AJ781003, CSHCG and CSW534983) (Hagen et al., 1993; Muller and Sackey, 2005). The product is 375 base pairs (bp) and runs from position 350 to $725 \mathrm{bp}$ on accession AJ608931. Both primers were manufactured by Sigma, UK as follows:

Forward primer: AACCTTGAGTACCTTGACCT

Reverse primer:

TCATTGACCAACCCACTGGTCAAG 
A master mix containing Taq polymerase and dNTPs was supplied by Qiagen (Multiplex PCR kit), UK. The PCR consisted of $5.0 \mu \mathrm{l} 2 \mathrm{x}$ master mix, $1.0 \mu \mathrm{l}$ primer solution containing $2 \mu \mathrm{M}$ of the CSSV forward and reverse primers, $3.0 \mu \mathrm{l}$ of water and $1.0 \mu \mathrm{l}$ of DNA and was run at $95^{\circ} \mathrm{C}$ for $15 \mathrm{~min}, 94^{\circ} \mathrm{C}$ for $30 \mathrm{sec}$, $56^{\circ} \mathrm{C}$ for $90 \mathrm{sec}, 72^{\circ} \mathrm{C}$ for $60 \mathrm{sec}$ at 35 cycles and with a final extension of $60^{\circ} \mathrm{C}$ for $30 \mathrm{~min}$. Unlabelled primers were used for the PCR/agarose electrophoresis while the forward primer was labelled with $\mathrm{HEX}^{\mathrm{TM}}$ for PCR/capillary electrophoresis. Fragment analysis of the PCR products was run on ABI P RISM 3100 Genetic Analyzer Capillary Sequencer.

Qualitative screening of CSSV: An internal control targeting a nuclear cocoa microsatellite was used in a multiplex PCR with the CSSV assay to differentiate between a successful and a failed PCR. The single copy microsatellite marker named mTcCIR25 (EMIL accession number Y16997) designed for Theobroma cacao by Lanaud et al. (1999) was used. The primer sequence for the marker is as follows: forward primer: CTTCGTAGTGAATGTAGGAG and reverse primer: TTAGGTAGGTAGGGTTATCT. The PCR protocol is the same as described with the addition of $\mathrm{mTcCIR} 25$ primers in the fragment analysis.

Sequencing of CSSV DNA: A PCR reaction was performed using DNA from the CSSV-infected
Amelonado cocoa tree and CSSV-infected genotype SCA 6 to verify that the primers selected targeted the CSSV genome correctly. The amplified product was cleaned using Nucleofast 96 plates (Nalgene, Macherey). For direct sequencing, $10 \mu \mathrm{l}$ volume reactions were performed containing $4 \mu \mathrm{l}$ Big dye and $1.6 \mu \mathrm{l}$ of individual primers $(1 \mu \mathrm{M})$ with $4.4 \mu \mathrm{l}$ of PCR products and centrifuged at $14,000 \mathrm{rpm}$ (SORVALL ${ }^{\circledR}$ Biofuge pico) for $10 \mathrm{~s}$. The thermo cycling conditions were: $96{ }^{\circ} \mathrm{C}$ for $30 \mathrm{~min}, 50{ }^{\circ} \mathrm{C}$ for $15 \mathrm{~min}, 60^{\circ} \mathrm{C}$ for $4.00 \mathrm{~min}$ and $10^{\circ} \mathrm{C}$ for $1 \mathrm{~min}$. The samples were sequenced using ABI Prism 3100 Genetic Analyzer Capillary Sequencer (16 capillary array model). The run was set up using the ABI Prism 3100 Genetic Analyzer Capillary Sequencer software version 1.0.1. The laser was an argon-ion laser with primary emission lines at $488 \mathrm{nM}$ and $514.5 \mathrm{nM}$.

The analysis was done with the DNA sequencing software Chromas version 2.0. The obtained sequence was compared with the CSSV genome available at GenBank (http://www.ncbi.nih.nlm.gov) and retrieved by BLAST searches. The alignment of the gene sequences was initially performed using CLUSTAL W (http://www.ebi.ac.uk/clustalw), subsequently inspected by eye and corrected manually.

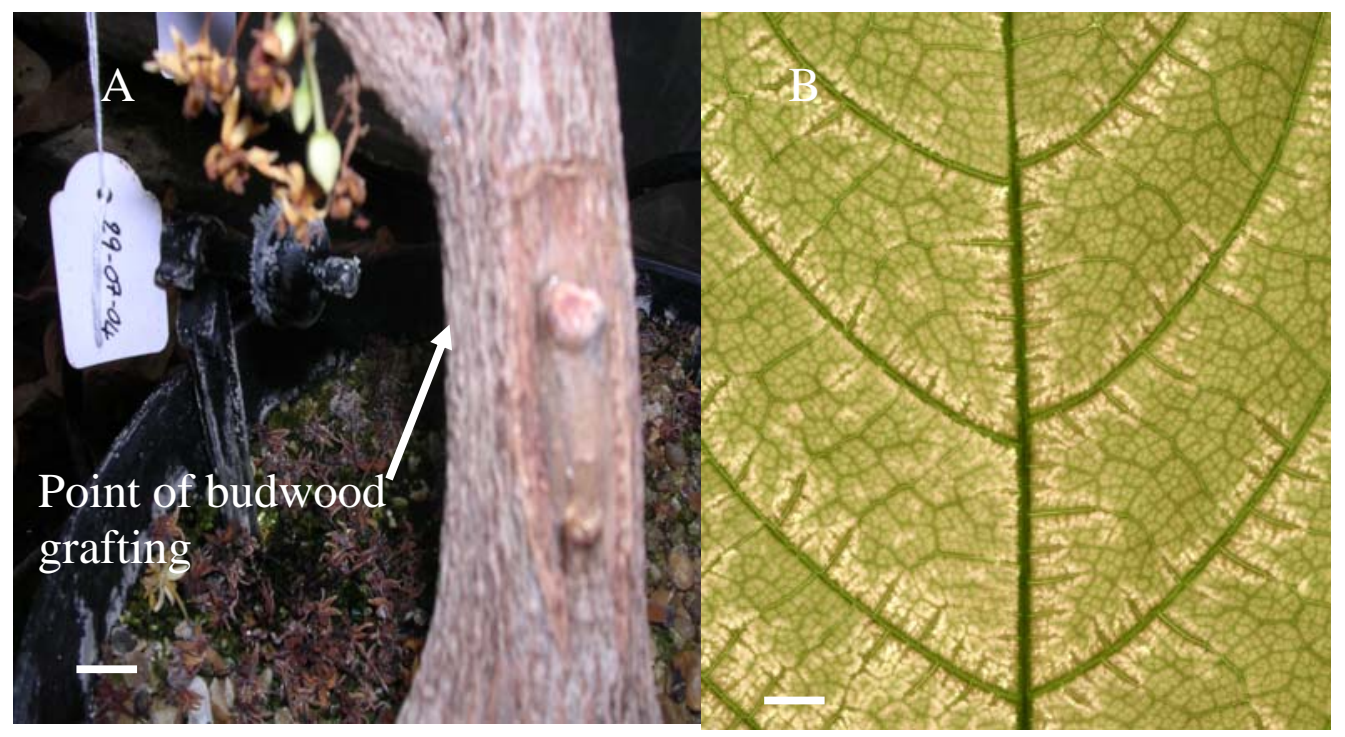

Fig 1 Budwood grafted cocoa clone and CSSV symptomatic leaf A Cocoa clone grafted with CSSV strain 1A-infected budwood, B Interveinal chlorotic CSSV symptoms 60 days post grafting. Scale bar $=1 \mathrm{~cm}$ 


\section{RESULTS}

Sequencing of CSSV: Primers for CSSV detection were designed from the alignment of the six published. CSSV sequences. The sequenced CSSV was identical to one of the Ghanaian CSSV strains (accession AJ7608931) (New Juaben) (Muller and Sackey, 2005) (figure 3).CSSV-infected Amelonado budwood grafted onto each of the cocoa clone (CC 222, CRU 100 and CL 19/10) became successfully established (Table 1).

Visual symptoms of CSSV were apparent in the new leaf flushes 110 and 116 (branch 1 and 2 respectively) days post grafting on CC 222 and 114 days post grafting on CRU 100 but 120 days post grafting no symptoms were detected on CL 19/10. Out of the nine somatic embryos derived plantlets on which budwood (from genotype SCA 6) were grafted only five became established (Table 2).

\begin{abstract}
Forward primer: aaccttgagtaccttgacct
CSHCG GAGTGGTATGAGAAGTCACACACAGCAAACCTTGAGTACCTTGACCTAGCATCTACTAGT 530 CSW534983 GAGTGGTATGAGAAATCACACACAGCAAACCTTGAGTACCTTGACCTAGCATCTACTAGT 531 AJ608931 GAATGGTATGAGAAGTCTCACACTGCCAACCTTGAGTACCTTGACCTAGCCTCTACCAGT 383 AJ609019 GAATGGTATGAGAAGTCTCACACTGCCAACCTTGAGTACCTTGACCTGGCCTCTACCAGT 385 AJ609020 GAATGGTATGAGAAGTCTCACACTGCCAACCTTGAGTACCTTGACCTAGCCTCTACCAGT 373 AJ781003 GAGTGGTATGAGAAGTCACACACAGCAAACCTTGAGTACCTTGACCTAGCATCTACTAGT 521

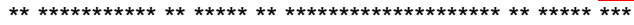

CSHCG AAAGTGACCAACAACCAGTTAGCACATAACCTTGCAGTAACCTTTGATAGAGTAAATTTA 590 CSW534983 AAAGTGACCAACAACCAGTTAGCACATAACCTTGCAGTAACCTTTGATAGAGTAAATTTA 591 AJ608931 AAAGTGACCAACAACCAACTAGCTCATAACCTCGCAGTAACCTTTGATAGAGTCAACTTA 443 AJ609019 AAAGTGACCAACAACCAACTAGCTCATAACCTCGCAGTAACCTTTGATAGAGTCAACTTA 445 AJ609020 AAAGTGACCAACAACCAACTAGCTCATAACCTCGCAGTAACCTTTGATAGAATCAACTTA 433 AJ781003 AAAGTGACCAACAACCAGTTAGCACATAACCTTGCAGTAACCTTTGATAGAGTAAATTTA 581
\end{abstract}

CSHCG GGTAACCGAGTTTTTATTAAAAACCTGAAGCAGATTCAAGAGTCTATTCTTGAATTAAAC 650 CSW534983 GGTAACCGAGTTCTTATTAAAAACCTGAAGCAGATTCAAGAGTCTATCCTTGAATTAAAC 651 AJ608931 GGAAACCGAGTCTTCATTAAAAACCTTAAACAAATCCAAGAGTCTATCCTAGAACTAAAT 503 AJ609019 GGAAACCGAGTCTTCATTAAAAACCTTAAACAAATTCAAGAGTCTATCCTAGAACTAAAT 505 AJ609020 GGAAACCGAGTCTTCATTAAAAACCTTAAACAGATTCAAGAGTCTATCCTAGAACTAAAT 493 AJ781003 GGTAACCGAGTTTTTATTAAAAACCTGAAGCAGATTCAAGAGTCTATTCTTGAATTAAAC 641 $* * * * * * * * * * * * * * * * * * * * * * * * * * * * * * * * * * * * * * * * * * * * * * * * * 10$

CSHCG ACCCGTGTTGATACTATAGAAGTAGCTCTAAGGAGGTTAACCAAGACCTTCAGAGAAAGC 710 CSW534983 ACCCGTGTTGATACTATAGAAGTAGCTCTAAGGAGGTTAACCAAGACCTTCAGAGAAAAC 711 AJ608931 ACCAGAATCGACACTGTAGAAGTAGCCTTGAAGAGGCTAACCAAGCAGTTCCGAGAAAAC 563 AJ609019 ACCAGAATTGACACTGTAGAAGTAGCCTTGAGAAGGCTAACCAAGCAGTTCCGAGAAAAC 565 AJ609020 ACCAGAATTGACACTGTAGAAGTAGCCTTGAGAAGGCTAACCAAGCAGTTCCGAGAAAAC 553 AJ781003 ACCCGTGTTGATACTATAGAAGCAGTTTTAAGAAGGTTAACCAAGACCTTCCGAGAAAAC 701 $* * * * * * * * * * * * * * * * * * * * * * * * * * * * * * * * * * * * * * * *$

CSHCG AAACCACTTACCGAAAGTGAAGTCCGTAAGTTGGTTGAAGAAATTGCTCAACAACCTAAG 770 CSW534983 AAACCACTTACCGAAAGTGAAGTCCGTAAGTTGGTTGAAGAAATTGCTCAACAACCTAAG 771 AJ608931 AAACCACTGTCTGAATCTGAAGTAAAGAGACTAGTAGAAGAGATAGCCCAGCAACCCAAA 623 AJ609019 AAACCACTGTCTGAATCTGAAGTAAAGAAACTAGTGGAAGAGATAGCCCAGCAACCCAAA 625 AJ609020 AAACCACTGTCTGAATCTGAAGTAAAGAGACTAGTGGAAGAGATAGCCCGGCAACCCAAA 613 AJ781003 AAACCACTTACCGAAAGTGAAGTCCGTAAGTTGGTTGAAGAAATTGCCCAACAACCTAAG 761

CSHCG ATCGTCGAGAAACAAGCACTCGAGATCTCTCAACAACTTGAGCAGAAACTAGAGAAGgTG 830 CSW534983 ATCGTCGAGAAACAAGCACTCGAGATCTCTCAACAACTTGAGCAGAAACTAGAAAAGGTG 831 AJ608931 ATTGTTGAGAAACAGGCACTGGAAATATCTCAACAGTTAGAACTCAAGTTAGAAAAAGTG 683 AJ609019 ATTGTTGAGAAACAGGCACTGGAAATTTCTCAACAGTTAGAACTCAAACTAGAAAAAGTG 685 AJ609020 ATTGTTGAGAAGCAGGCACTGGAAATATCTCAACAATTAGAACTCAAACTAGAAAAAGTG 673 AJ781003 ATCGTCGAGAAACAAGCACTCGAGATCTCTCAACAGCTTGAGCAGAAGCTGGAGAAAGTT 821 $* * * * * * * * * * * * * * * * * * * * * * * * * * * * * * * * * * * * * * * * *$

Reverse primer: tcattgaccaacccactggtcaag

CSHCG GAAAAGCTCTTGCACAAACTTGACCAGTGGGTTGGTCAATGACTGATAGCCCAGCCTATC 890 CSW534983 GAAAAGCTCTTGCACAAACTTGACCAGTGGGTTGGTCAATGACTGATAGCCCAGCCTATC 891 AJ608931 GAAAAACTTCTACACAAGCTTGACCAGTGGGTTGGTCAATGAGTGAAAGTCCCTCTTACC 743 AJ609019 GAAAAGCTTCTACACAAGCTTGACCAGTGGGTTGGTCAATGAGTGAAAGCCCCTCTTACC 745 AJ609020 GAAAAGCTTCTACACAAGCTTGACCAGTGGGTTGGTCAATGAGTGAAAGCCCCTCTTACC 733 AJ781003 GAAAAACTTCTCCATAAGCTTGACCAGTGGGTTGGTCAATGACCGACAGCCCGTCTTACC 881

Fig 2 CSSV genomic sequences indicating the positions of the forward and reverse primers. 
Agric. Biol. J. N. Am., 2010, 1(6): 1357-1364

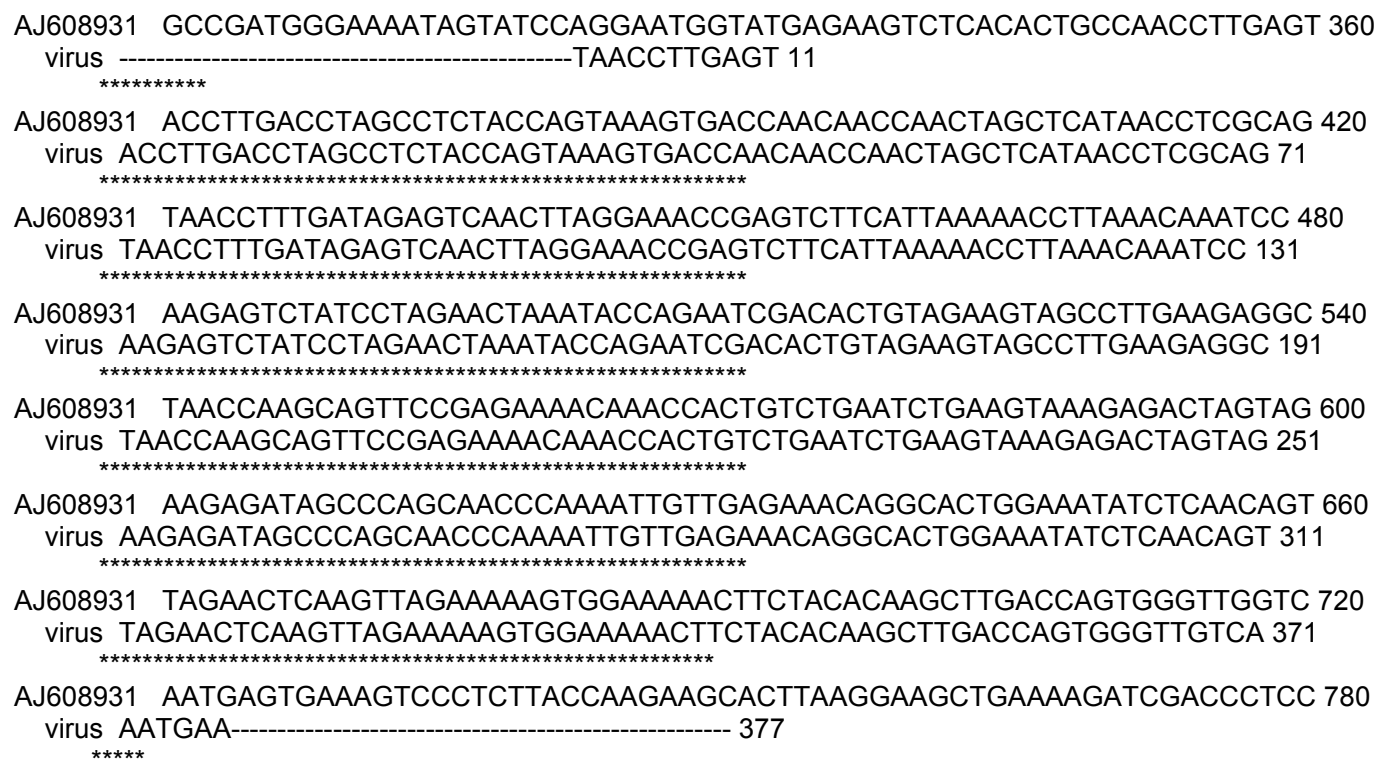

Fig 3 CLUSTAL W (1.83) multiple sequence alignment of CSSV strain 1A in infected cocoa materials.

CSSV was detected in branch 1 of samples $2,4,6$ and 9 between 95 to 165 days post grafting and branch 2 of samples 3, 4 and 9 between 95 to 179 days post grafting. CSSV leaf symptoms were detected in branch 1 of samples 4, 6 and 9 between 145 to 165 days post grafting and branch 2 between 173 to 179 days post grafting

\section{DISCUSSION}

Limitations of grafting and budwoodding: There are limitations to the successful grafting and budwooding of two different plants as the botanical relationship between two plants is not a guarantee of a successful union (Dirr and Heuser, 2006). Different species within the same genus are compatible in some instances but incompatible in others. Incompatibility may be expressed in a number of ways including combinations which never form a successful union and types in which the union is successful initially, the scion initially successfully develop into a branch and later dies or the plant eventually dies (Dirr and Heuser, 2006). Usually, in a successful union in cocoa the scion remain green and firmly attached to the stock (Figure 1), while the scion looks dark and shrinks in a failed union (Quainoo, 2006).

Budwood graft transmission of CSSV in cocoa clones: Cocoa clones CC 222, CRU 100 and CL 19/10 grafted with CSSV-infected Amelonado budwood (T2) demonstrated the movement of virus to the new leaf flushes. PCR/capillary electrophoresis revealed the presence of virus (in genotype CC 222 and CRU 100) 90 days post grafting respectively and 120 days post grafting for CL 19/10 (Table 1). Speed of CSSV movement over an average distance of 40 $\mathrm{cm}$ in the multiple leaf point samples above the point of grafting was consistently greater in genotypes CC 222 and CRU 100 than genotype CL 19/10. Assuming all the cocoa clones were of the same age, equal heights and sizes, this differences in the speed of transmission of the virus may be attributed to genotype differences.

Budwood graft transmission of CSSV in cocoa plantlets: Amelonado cocoa trees readily express visual symptoms of CSSV if infected (for all virus strains). Usually, in quarantine practices for the indexing of CSSV, donor budwoods are grafted to disease-free Amelonado cocoa trees which express visual symptoms of CSSV within the new leaf flushes. CSSV latent infections however may take up to two years to be expressed on the new leaf flushes which make visual indexing for CSSV inadequate. Other cocoa clones or genotypes may not express visual symptoms of the virus in the new leaf flushes when CSSV-infected budwoods other than Amelonado are grafted on them. Even when visual symptoms are expressed, they occur in the first few leaf flushes and then vanish (Quainoo, 2006). 
Agric. Biol. J. N. Am., 2010, 1(6): 1357-1364

Table 1 PCR-based detection of CSSV in graft-infected cocoa clones

\section{Cocoa clone 1 \\ Cocoa clone 2}

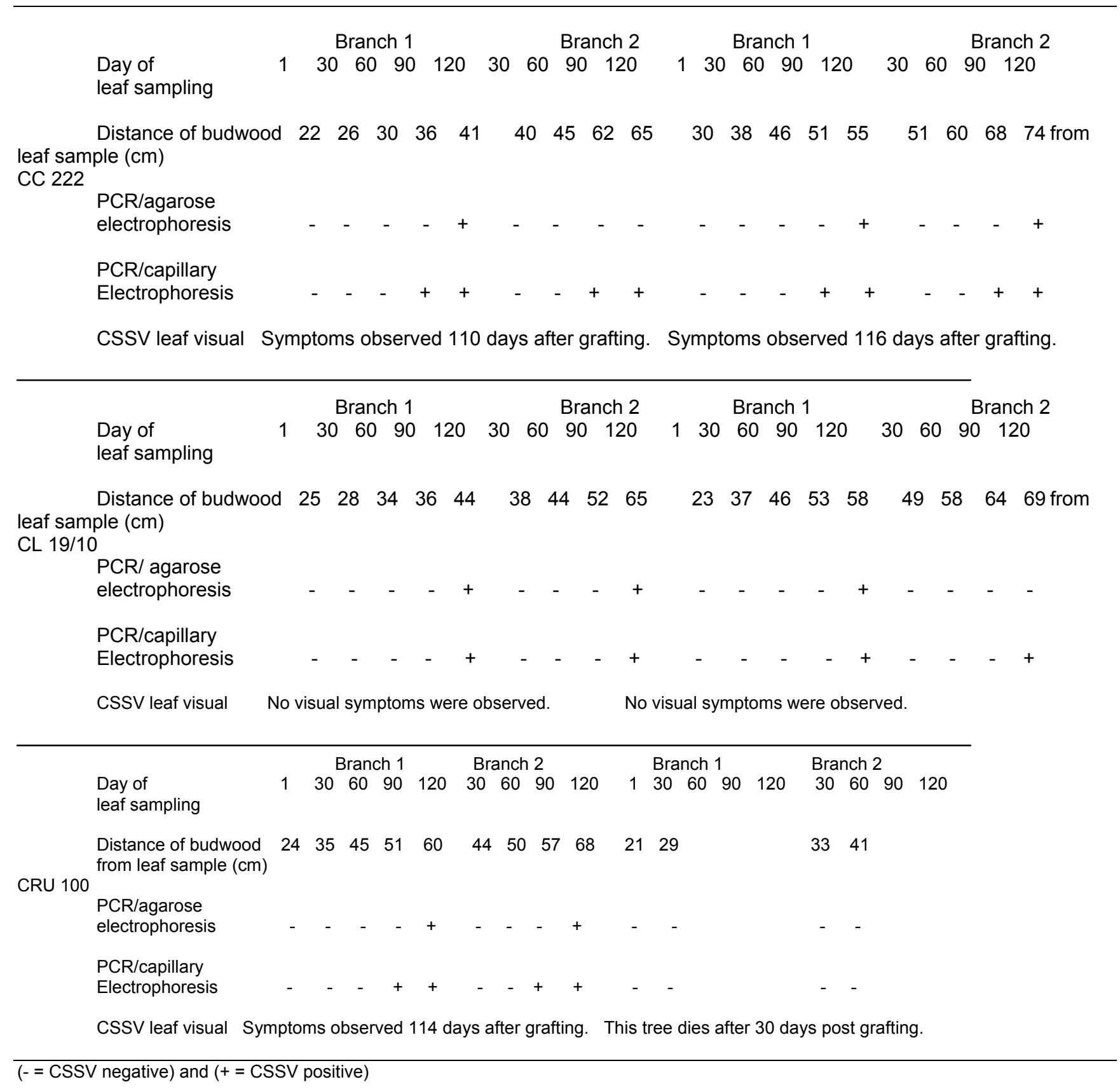


Agric. Biol. J. N. Am., 2010, 1(6): 1357-1364

Table 2 PCR-based detection of CSSV in graft-infected cocoa plantlets up to six months

$\begin{array}{ccc}\text { Detection of CSSV in leaves by } & \text { Detection of CSSV leaf } \\ \text { PCR/capillary electrophoresis } & \text { symptoms (days post grafting) }\end{array}$

(days post grafting)

Plantlets

Branch $1 \quad$ Branch 2

Branch 1

Branch 2

$1=\mathrm{AMAZ12}$

$2=\mathrm{EET} 272$

$3=\mathrm{EET} 272$

$4=I M C 14$

$5=$ LCTEEN $16 / 100$

$6=\mathrm{SCA} 3$

$7=\mathrm{SCA} 6$

$8=$ SPA 4

$9=$ SPA 4

\section{5}

145

$-$

165

$-$

95

-
-
179
-
-
-
-
165

165

$\begin{array}{ll}- & - \\ - & - \\ - & 173 \\ 165 & 179 \\ - & - \\ 165 & - \\ - & - \\ - & - \\ - & -\end{array}$

(- = CSSV negative)

Failure of four budwood grafted plantlets to establish union with plantlets does not mean the CSSV was not exported into the plantlets tissues. This is supported by the observation of transient visual symptoms on plantlet 4 (IMC 14) (the graft failed to establish) 165 and 179 days post grafting for branches one and two respectively, and further confirmed by PCR/capillary electrophoresis (Table 2). Furthermore, plantlet 2 (EET 272) (the graft failed to establish) 165 days post grafting did not revealed visual symptoms but viral presence were confirmed by PCR/capillary electrophoresis. A possible explanation for this is that the CSSV-infected budwood lined up with the cambium layer of the plantlets resulting in the transmission of the virus into the plantlets (Table 2). The results suggested that the success or failure of budwood grafting is largely irrelevant for the transmission of the CSSV once contact between cambium layers in the union is established. Visual symptoms of CSSV are therefore not guaranteed in a successful budwood grafting.

Usually, in cocoa quarantine procedures and germplasm distribution two years of leaf inspection of cocoa genotypes for visual symptoms of CSSV is carried out before they are certified to be viral free if no symptoms are observed. Detection of CSSV in all plantlets without visual symptoms (Table 2) suggested that the primers for the PCR/capillary electrophoresis was robust enough to detect the virus well in advance before the establishment of visual symptoms. This suggested that visual inspection alone is inadequate for the detection of CSSV and that the PCR primer based procedure though expensive is more reliable and quicker in detecting the virus. The design of the primers across the six published sequences of the cocoa genome (figure 2) capable of detecting all strains of the CSSV has the potential of improving the robustness of quarantine procedures for the international exchange and long term conservation of cocoa germplasm.

\section{CONCLUSION}

The primer designed from the published sequences of cocoa genome was capable of detecting CSSV strain 1A in the infected cocoa experimntal materials. $\mathrm{PCR} /$ capillary electrophoresis highlighted the virus well in advance before the appearance of visual symptoms. The PCR-based procedure for screening of cocoa materials for CSSV is more reliable than visual expression of the virus on infected trees. This has significant implications for international exchange and conservation of cocoa germplasm.

\section{ACKNOWLEDGEMENTS:}

This work was supported by a scholarship from the Ghana government. A. K. Quainoo is grateful to $\mathrm{Dr} A$. C. Wetten for supervising his Ph.D research and $\mathrm{Dr}$ Joel Allainguillaume who assisted him with the molecular aspect of this work. Thanks to Professor Paul Hadley for the use of facilities at the University of Reading Cocoa Intermediate Quarantine Unit.

\section{REFERENCES}

Cilas, C., Muller, E. and Mississo, E (2005). Plant disease, 89 (8) 913.

Dirr, M. A. and Heuser, C. W (2006). "From the ground up" grafting and budding. Website of American Bonsai Society. Http:www.absbonsai.org/articles/groundup/grafting.htm

Frison, E. A.: Diekman, M. and Nowell, D (1999). FAO/IBPGR Technical Guidelines for the safe 
movement of germplasm No. 20. Cacao ( $1^{\text {st }}$ revision). Food and Agriculture Organization of the United Nations, Rome/International Plant Genetic Resources Institute, Rome.

Hagen, L. S.; Jacquemond, M.; Lepingle, A.; Lot $H$. and Tepfer, M. (1993). Nucleotide sequence and genomic organization of cacao swollen shoot virus. Virology, 196:619-628.

Lanaud, C.; Risterucci, A.M.; Pieretti, I.; Falque, M.; Bouet, A. and Lagoda, P. J. L (1999). Isolation and characterization of mcrosatellites in Theobroma cacao L. Molecular Ecology, 8:2141-2152.

Leg, J. T (1972). Measures to control the spread of cocoa swollen shoot virus in Ghana. PANS, 18:1:57-60

Muller, E (2008). In: Rao Govind P. (ed.), Paul Khurana S.M. (ed.), Lenardon Sergio L. (ed.). Characterization, diagnosis and management of plant viruses. Industrial crops. Houston: Stadium Press LLC, pp. 423-444.

Muller, E. and Sackey, S (2005). Molecular variability analysis of five new complete cacao swollen shoot virus genomic sequences. Arch Virol, 150:53-66

Owusu, G. K. and Bonney, J. K (1972). Virus research: studies of CSSV isolates from Ashanti Region. Report of the Cocoa Research Institute of Ghana for 196970:63-64

Posnette, A. F. (1947). Virus diseases of cacao in West Africa. 1. Cacao viruses $1 \mathrm{~A}, 1 \mathrm{~B}, 1 \mathrm{C}$ and 1D. Annals of Applied Biol, 34(3): 388-402.

Posnette. A. F. (1944). Swollen shoot in Trinidad. Tropical Agriculture (Trinidad), 21:105.

Quainoo, A.K (2006). Germplasm conservation of cocoa (Theobroma cacao L.) and virus elimination through tissue culture. PhD Thesis, School of Biological Sciences, University of Reading. 192 pages.

Quainoo, A.K.; Wetten, A. C. and Allainguillaume, J (2008a). Transmission of cocoa swollen shoot virus by seeds. J Virological Methods, $150: 45-49$.

Quainoo, A.K.; Wetten, A. C.and Allainguillaume, J (2008b). The effectiveness of somatic embryogenesis in eliminating the cocoa swollen shoot virus from infected cocoa trees. J Virol Methods, 149(1): 91-96.

Thresh, M. J (1980). The origin and epidemiology of some important plant virus diseases. Applied Biol, 5: 1-65.

Thresh, M. J (1958). The spread of virus disease in cacao. West African Cacao Research Institute Technical Bulletin No. 5. 